\title{
L'évolution didactique du FLE en Espagne et de l'ELE en France : cheminements singuliers et distincts
}

\author{
Alexandra Marti ${ }^{1}$
}

Recibido: 24 de octubre de 2017 / Aceptado: 23 de julio de 2018

Résumé. Si les sigles FLE (Français Langue Etrangère) et ELE (Espagnol Langue Etrangère) font dorénavant partie du vocabulaire universitaire aussi bien en France qu'en Espagne, sait-on vraiment leur signification? Il s'agira de réviser dans ce travail la notion de champ propre au FLE et à l'ELE en didactique et de montrer qu'il est très difficile de définir avec précision la succession chronologique des méthodologies en présence pour une langue en particulier, étant donné que certaines d'entre elles ont cohabité avant de s'imposer aux précédentes et d'autres n'ont même pas eu le temps de s'établir ni même de se développer. Les évolutions didactiques du FLE en Espagne et de l'ELE en France en sont un exemple. Il est donc important de les évoquer sommairement pour mieux comprendre les méthodologies qui prédominent actuellement et qui sont semblables de part et d'autre des Pyrénées. Pourtant, si le point de départ est le même (méthodologie traditionnelle) et le point d'arrivée aussi (perspective actionnelle), l'évolution didactique du FLE en Espagne et de l'ELE en France diffère considérablement, d'où l'intérêt de rendre compte des similitudes et divergences dans ce travail.

Mots-clés : FLE; ELE; Didactique des Langues Etrangères (DLE); méthodologie; évolution

\section{[en] The Didactic Evolution of FFL in Spain and SFL in France : Singular and Distinct Paths}

\begin{abstract}
If the acronyms FFL (French as a Foreign Language) and SFL (Spanish as a Foreign Language) are now part of the university vocabulary both in France and Spain, does one really know their meaning? It will be necessary to review in this work the notion of field specific to the FFL and the SFL in didactics and to show that it is very difficult to define precisely the chronological succession of the methodologies for a particular language, since some of them coexisted before imposing themselves on the former ones and also that others did not even have the time to establish nor even to develop. The didactic evolutions of the FFL in Spain and the SFL in France are an example. It is therefore important to summarize them in order to understand better the methodologies that currently predominate and which are similar on both sides of the Pyrenees. However, if the starting stage is the same (with the traditional methodology) and the ending stage is the same (with the actional perspective), the didactic evolution of the FFL in Spain and the SFL in France differs considerably to account for these similarities and divergences in this work.
\end{abstract}

Keywords: FFL; SFL; Foreign Languages Didactics (DLE); methodology; evolution

Sommaire. 1. Introduction. 2. Le champ du FLE en didactique. 3. Le champ de l'ELE en didactique. 4. Les domaines du FLE et de l'ELE comme champs spécifiques. 5. Le FLE en Espagne. 5.1 L'évolution

Universidad de Alicante / Université Paris V Descartes. Departamento de Filologías Integradas

E-mail : alexandra.marti@ua.es 
méthodologique progressive. 5.2 L'influence française. 6. L'ELE en France. 6.1 L'évolution méthodologique atypique. 6.2 L' « ellipse méthodologique ». 7. Conclusion.

Cómo citar: Marti, A. (2018).L'évolution didactique du FLE en Espagne et de l'ELE en France : cheminements singuliers et distincts, en Revista de Filología Románica 35, 225-239.

\section{Introduction}

La Didactique des Langues Etrangères (DLE), au sens large, est jalonnée d'une succession de méthodologies en constante évolution dont certaines ont été perçues comme des innovations, prédominant sur des périodes relativement longues. C'est ainsi qu'à la méthodologie traditionnelle du XIX ${ }^{\mathrm{e}}$ siècle ont succédé différents courants méthodologiques, dont les plus évidents et incontestables ont été : la méthodologie dite « directe », entre 1900 et 1920, « active », entre les années 1920 et 1930 jusqu'en 1960 dans l'Enseignement scolaire français des LE (Puren, 1988), audio-orale (MAO), entre les années 1940 et 1960, audio-visuelle (MAV) puis structuro-globale audio-visuelle (SGAV) de 1960 à la fin des années 70. Dans les années 1975, l'approche communicative marque un tournant important jusqu'au début des années 2000. De nos jours, c'est une approche dite « actionnelle » promue par le Conseil de l'Europe et le Cadre Européen Commun de Référence pour les Langues-CECRL (Barthélémy, Groux, Porcher, 2013). De manière simplifiée, ce sont les méthodologies les plus importantes qui ont marqué l'évolution de la DLE.

Pourtant cette présentation panoramique, quelque peu réductrice de l'histoire des méthodologies, est très discutable car c'est un schéma classique, se présentant comme un cadre commun pour l'ensemble des langues, comme s'il s'agissait d'une évolution linéaire : les méthodologies se remplaçant les unes des autres, les unes en rupture avec les précédentes, les autres comme une adaptation de ces dernières, ce qui n'est évidemment pas aussi systématique (Rodríguez Seara, 2001). Cela s'explique certainement par le fait que l'évolution didactique des langues, dans un contexte particulier, est spécifique et unique, et ne suit pas toujours les périodes ni les grandes lignes des méthodologies de la DLE. Cette étude se centrera sur l'évolution didactique du FLE en Espagne et de l'ELE en France, deux champs spécifiques, ayant des cheminements singuliers et distincts.

\section{Le champ du FLE en didactique}

Du côté de l'Hexagone, le FLE garde, selon Cuq et Gruca, " une part de mystère » car du mariage Français et Langue Étrangère est né :

un être académique curieux, à qui il a bien fallu faire une (petite) place dans la famille, sans être vraiment sûr qu'il en soit digne. Comme tous les enfants dont on suspecte un peu les origines, il éprouve un fort besoin de prouver sa légitimité. Comme tous les enfants issus de cultures différentes, il a plus ou moins conscience de représenter un prototype d'avenir (Cuq et Gruca, $2003: 13$ ). 
Les auteurs ont bien conscience de ce besoin pour le FLE de prouver sa légitimité et leur travail œuvre dans ce sens. Ils expliquent que le français, en tant que LE, s'est doté petit à petit d'un arsenal de moyens destinés à faciliter son appropriation par des apprenants étrangers. Ce faisant, il s'est ancré dans le domaine de l'action et de la réflexion, communément appelé : la didactique des langues, qui s'intéresse principalement à l'appropriation d'une langue par le processus d'enseignement/apprentissage (E/A), bien qu'elle analyse aussi l'appropriation des langues en milieu naturel. Pour Cuq et Gruca (2003 : 22), le FLE peut se décrire à partir d'une structuration synchronique sous divers aspects à la fois : géolinguistique, politique, linguistique, culturel, etc. En tant qu'objet de réflexion didactique, il doit s'armer d'un outillage conceptuel efficace. Les chercheurs l'abordent ici selon le concept de «champ ».

Ce concept, provenant du sociologue Bourdieu (1979), a été méticuleusement explicité et introduit par Porcher (1987) dans la didactique du FLE impliquant des agents (individus, organismes, institutions) qui partagent un intérêt commun (la diffusion du FLE), avec ses enjeux propres (rémunérations, distinctions etc). La didactique du FLE n'échappe en rien à cette « logique de champ » comme le montrent Cuq et Gruca (2003 : 23):

Pour la didactique du FLE, le champ constitue un concept de niveau 1, c'est-à-dire un concept englobant, à l'intérieur duquel s'organisent des concepts opératoires de niveaux inférieurs. Le champ du FLE est un système, c'est-à-dire un ensemble structuré d'éléments et de relations. L'objet de la didactique est d'une part de décrire le système, mais d'autre part aussi de le développer et donc de contribuer à sa modification.

Selon Barthélémy (2007), le terme FLE a fait son apparition après la Seconde Guerre Mondiale, au fur et à mesure de la création d'un réseau de diffusion de la langue et de la culture françaises à travers le monde, pour lutter notamment contre la suprématie de la langue anglaise. Il s'agit là d'un véritable champ disciplinaire qui rassemble actuellement des acteurs partageant le même objectif : développer et diffuser la langue française. Ce concept fait donc référence au français hors frontières, bien qu'il s'utilise également en France.

Alors qu'elle recouvre généralement des situations d'enseignement/apprentissage $\mathrm{du}$ français hors de nos frontières, cette terminologie s'applique aussi dans l'Hexagone à des dispositifs de moins en moins particuliers (formation des étudiants étrangers dans les universités, migrants, classes d'accueil...) : le professeur de FLE enseigne le français à des non-francophones, et ce, pas seulement hors de nos frontières (Barthélémy, 2007 : 77).

L'histoire du FLE comme langue d'enseignement à l'étranger et/ou pour étrangers est loin d'être récente. Son évolution en tant que champ propre a dû subir un processus d'institutionnalisation. En FLE, il a débuté par la fondation de l'Alliance française (1883) et de la Mission laïque (1902), puis du CREDIF (Centre de Recherche et d'Etudes pour la Diffusion du Français) et du BELC (Bureau d'Études pour la Langue et la Civilisation Françaises à l'Étranger). Ces deux centres spécialisés occuperont une place fondamentale dans le développement du FLE, le légitimant 
en tant que champ. Ce sont d'ailleurs ces deux institutions qui sont à l'origine des instances de légitimation actuelles.

L'année 1961 voit apparaître Le Français dans le monde, première revue consacrée intégralement à l'enseignement du FLE à l'étranger, ce qui constituera aussi un tournant capital dans le processus de légitimation de ce domaine. Les maisons d'édition permettront également de renforcer cette discipline. Pourtant, au niveau de l'Enseignement supérieur, le FLE peine à s'imposer. Comme souligne Barthélémy (2007: 11) :

Mais point de légitimation sans reconnaissance universitaire. Il faudra attendre 1983 pour que, sous la houlette de Louis Porcher, une première commission de spécialistes propose la maquette de diplômes nationaux spécifiques à la formation d'enseignants de FLE et pour qu'une seconde débouche sur la mise en place, à la rentrée 1985, de certifications en français pour étrangers, nécessaires dans le contexte de compétition que se livrent les langues - diplômes élémentaire et approfondi de langue française.

L'année 1983 est donc charnière pour le FLE et c'est à ce moment-là que les cursus universitaires en France incorporent la formation des enseignants de FLE qui était, jusque dans les années 80 , dispensée par les organismes cités précédemment. La création de filières et de diplômes universitaires légitime ainsi ce champ disciplinaire.

\section{Le champ de l'ELE en didactique}

En Espagne l'ELE (Espagnol Langue Etrangère) représente, contrairement à la France, un champ relativement récent dans la mesure où cette discipline commence à être reconnue dans le milieu universitaire espagnol. Ce manque de reconnaissance peut s'expliquer surtout, comme l'indique Cestero Mancera (2006 : 14) par le fait d'avoir considéré cette discipline comme peu prestigieuse depuis de nombreuses années : « Hasta hace relativamente pocos años, dar clases de español para extranjeros se consideraba, podemos decir, una actividad poco honrosa para un filólogo del español, y se dejaba este tipo de trabajo para los especialistas de otras filologías ». Cette situation a évidemment changé et, maintenant, nombreux sont les hispanistes, spécialistes en langue et culture espagnoles, qui souhaitent mieux connaître la méthodologie de l'enseignement des LE, ouvrant ainsi un vaste champ de travail et de réflexion.

L'Institut Cervantes a d'ailleurs déclaré l'année 2009 comme charnière symbolisant la célébration du $25^{\mathrm{e}}$ Anniversaire de la naissance de la spécialité ELE et, de nos jours, elle est devenue un champ disciplinaire qui s'est constitué lentement mais sûrement dans le milieu universitaire espagnol, uni désormais par un enjeu commun (la diffusion de la langue et de la culture espagnoles), des acteurs luttant pour leurs positions, une administration centrale, des instruments d'actions (les manuels d'enseignement de l'espagnol), des livres de références, des revues, des sites, des collections d'ouvrages. Comme l'explique Cassany (2009:2) : 
En estos 25 años el mundo de ELE ha crecido como un adolescente y se ha presentado en sociedad como una joven que asiste a su primer baile. Se ha consolidado como necesidad e interés social, mercado económico, disciplina profesional y campo de investigación; se ha articulado en instituciones, asociaciones, editoriales específicas, congresos, planes curriculares, certificaciones reconocidas, materiales didácticos para muchos contextos, ofertas de formación inicial y continuada de profesorado. ¡Ya es toda una señorita bella y coqueta!, conocida, reconocida y admirada por casi toda la sociedad...

C'est aussi un domaine universitaire propre à l'Espagne, avec ses diplômes nationaux de deuxième et troisième cycles, ses masters et ses doctorats. Cela concerne évidemment ses emplois spécifiques, dotés d'une légitimation reconnue. De très nombreux étudiants sont concernés chaque année, espagnols et étrangers, pour lesquels des centres de recherche officiels avec leurs chercheurs professionnels d'ELE, leurs publications scientifiques et leurs échanges internationaux sont désormais accessibles. Les enseignants d'ELE en Espagne reçoivent aujourd'hui une formation professionnelle de grande qualité qui attire un nombre de plus en plus important d'étudiants et de candidats.

\section{Les domaines du FLE et de l'ELE comme champs spécifiques}

En France, à part la discipline du FLE, on peut constater que celle de l'ELE, en tant que nouveau champ d'investigation et nouvelle matière à enseigner à l'université pour la formation de futurs professeurs de LE, est presque inexistante. Il y a certes des départements d'études hispaniques mais l'enseignement imparti renvoie à un espagnol strictement académique, celui des grands auteurs de la littérature espagnole et hispano-américaine. Sans oublier les exercices de traduction (thème/version) toujours d'actualité dans le système universitaire français. L'intérêt pratique de l'enseignement de la langue peine à se développer même pour les non spécialistes, contraints eux aussi, la plupart du temps, d'apprendre un espagnol purement académique.

Il ne serait donc pas absurde de réfléchir aux conditions dans lesquelles pourraient être instauré un Master ELE en France, qui donnerait un label officiel aux compétences linguistiques et pédagogiques acquises, et qui, surtout, fournirait les moyens d'articuler l'enseignement de l'ELE en France. Même si, actuellement, il existe le Master "Métiers de l'Enseignement, de l'Éducation et de la Formation » (MEEF), pour préparer les futurs enseignants d'espagnol à exercer en France, il serait intéressant de proposer un master d'ELE dans les universités françaises, comme l'actuel master FLE en France, en tant que nouveau champs d'investigation, permettant aux étudiants français d'enseigner l'espagnol dans de nombreux pays étrangers.

Reste donc à s'interroger sur l'avenir de l'ELE, champ relativement nouveau qui s'avère prometteur à l'étranger et en Espagne même, où les étrangers sont de plus en plus nombreux à étudier, comme l'attestent les nombreux programmes d'échanges interuniversitaires.

En somme, de nos jours, les domaines du FLE et de l'ELE comme champs spécifiques dans l'enseignement des LE sont absolument nécessaires, en raison d'une forte diversification universitaire et du développement accru de nouvelles méthodes 
permettant aux étudiants d'acquérir des compétences aussi bien linguistiques que culturelles.

Par ailleurs, même si les hautes sphères de l'Union Européenne reconnaissent incontestablement la prédominance de l'anglais dans les secteurs-clés de la société comme l'économie, le commerce, l'informatique, le tourisme et les relations internationales, il s'avère que la langue anglaise seule ne suffit pas à l'Europe pour gérer la concurrence économique mondiale ni pour préserver la diversité culturelle. C'est la raison pour laquelle pour rééquilibrer cette situation il incombe aux institutions académiques de promouvoir la diffusion de plusieurs langues, comme par exemple le FLE et l'ELE. La diversification entraîne alors l'assurance de faire vivre toutes les langues, des plus minoritaires aux plus connues :

L'enseignement des langues vivantes dans les systèmes éducatifs des Etats-membres du Conseil de l'Europe doit, par conséquent, être davantage diversifié. Il doit se traduire par l'acquisition non seulement de l'anglais, mais aussi d'autres langues européennes et mondiales par tous les citoyens européens, en parallèle avec la maîtrise de leur langue nationale et, le cas échéant, régionale (Conseil de l'Europe, 1998: recommandation 1383).

Ce faisant, l'Assemblée parlementaire du Conseil de l'Europe promeut la diversification linguistique de l'Europe en soutenant qu'elle « constitue un riche patrimoine culturel qu'il faut sauvegarder et protéger » (recommandation 1383). D'où l'importance de promouvoir et de préserver le FLE et l'ELE, véritables champs spécifiques, comme il l'a été démontré, avec leurs enjeux (la promotion de la langue et de la culture espagnoles et françaises), leurs acteurs (administrateurs, formateurs, chercheurs, et professeurs), leurs institutions de régulation (bureaucratiques : Ministères des Affaires étrangères, Ministerio de la Educación, Cultura y Deporte, Ministère de l'Education Nationale, institutions académiques françaises et espagnoles), leurs outils de diffusion (ambassades à l'étranger, Instituto Cervantes, Alliance Française, le Conseil de l'Europe, le Cadre Européen Commun de Référence pour les Langues, le programme Erasmus...), et leurs luttes de position entre les différents partenaires.

Cela suppose le défi de lancer la diffusion du FLE et de l'ELE à l'échelle nationale pour justement impulser leur enseignement à l'étranger et éviter ainsi l'uniformisation anglophone.

\section{Le FLE en Espagne}

En dépit de la tradition séculaire de l'enseignement du FLE en Espagne, de l'abondante production des méthodes de français aussi diverses les unes que les autres, on ne manque pas de constater l'existence de recherches fortes intéressantes qui ont été réalisées sur l'évolution didactique de la langue française en Espagne.

Les principales investigations font notamment référence aux thèses de Calle Carabias (1990), Fernández Fraile (1995), Morales Gil (2003), Urbano Marchi (2003) etc. ainsi qu'aux recherches de Fernandez Fraile et Suso López (1999), Suso López (2005), García Bascuñana (2005, 2008), Lépinette (1997, 2000, 2006, etc.), Morales Gil $(2007,2009)$. Cela fait donc plus de 25 ans approximativement qu'un intérêt cer- 
tain se manifeste en faveur de la recherche dans le domaine de l'histoire de l'enseignement des langues. Les publications de la Société Internationale pour 1' "Histoire du Français Langue Étrangère ou Seconde » (SIHFLES) en sont la preuve.

\subsection{L'évolution méthodologique progressive}

Loin de faire une étude exhaustive du cheminement du FLE en Espagne, il s'agira ici de montrer succinctement l'évolution didactique du français dans ce pays (à partir du début du XIX ${ }^{\mathrm{e}}$ siècle jusqu'à l'actualité). C'est ainsi que s'ouvre en Espagne (fin $\mathrm{XVIII}^{\mathrm{e}}$ - début $\mathrm{XIX}^{\mathrm{e}}$ siècle) une longue période dominée par la méthodologie traditionnelle dont il est souvent bien difficile de dresser le bilan exact tant son évolution est ample dans le temps. Fondée sur l'enseignement de règles grammaticales, la traduction et la prédominance de l'écrit littéraire, cette méthodologie appartient désormais à la préhistoire de la didactique du FLE. Les professeurs enseignaient les langues comme le latin ou le grec c'est-à-dire comme des langues mortes, et les élèves recouraient à la LM et à la traduction.

Après cette méthodologie archaïque où la rigidité des méthodes avait pour norme la langue des grands auteurs qu'il fallait imiter, la méthodologie directe qui s'était déjà imposée en Allemagne et plus tard en France pour s'opposer à la précédente, perçue comme obsolète et inefficace, commence à apparaître en Espagne.

Comme l'explique García Bascuñana (2005 : 140) :

Contra esa situación, que mantiene la enseñanza de las lenguas vivas en un estado de precariedad y de rutina evidente - al margen de las nuevas corrientes metodológicas-, reaccionarán ciertas instituciones y centros al margen de la enseñanza oficial, sobre todo a partir de las aportaciones del método directo que se impone definitivamente en Alemania y posteriormente en Francia hacia 1870-1880, y que en países como España empieza a contar con cartas de naturaleza de una manera más o menos declarada con el cambio de siglo.

Au niveau international, la diffusion de la méthodologie directe représentera une évolution importante dans l'enseignement, dont la principale originalité sera le recours à la LE dès le début de l'apprentissage, en s'interdisant d'utiliser la LM et en favorisant la transmission d'un vocabulaire courant, d'une grammaire inductive, l'acquisition de l'oral et, par conséquent, une bonne prononciation. Toutefois, comme l'évoque Fernández Fraile (1995), la méthodologie directe a eu une très faible répercussion en Espagne et malgré les recommandations du Ministère en faveur d'un enseignement plus pratique, le corps enseignant s'était habitué à une conception formative et culturelle fondée sur des méthodes traditionnelles dépourvues d'un quelconque objectif utilitaire de la langue. En conséquence, très peu d'enseignants avaient osé mettre en pratique dans leurs classes la méthode directe.

Par ailleurs, une autre méthodologie, trop souvent oubliée mais ayant eu un impact dans la didactique du FLE en Espagne, sera celle de la méthodologie active qui apparaîtra principalement après la Première Guerre Mondiale. Ainsi que le mentionne Morales Gil (2007), la méthodologie active occupera au moins une dizaine d'années en Espagne, à partir des années 50 du XX ${ }^{\mathrm{e}}$ siècle et même au-delà (1957-1967), lorsque 
l'Espagne commencera à s'ouvrir aux influences étrangères, moment à partir duquel une augmentation progressive et imparable de l'intérêt pour l'apprentissage des langues vivantes (LV) est observée dans de nombreux pays. C'est précisément en août 1953, convoqué et organisé par l'UNESCO, qu'a lieu à Nuwara Eliya un Congrès International consacré à l'enseignement des LE. Ce congrès, qui a duré quatre semaines, était consacré à «La contribution de l'enseignement des $L V$ à 1'éducation pour le civisme international » et a réuni une quarantaine de spécialistes venus de 18 pays différents. La France a joué un rôle de premier plan, puisque le sous-comité des LV de la Commission de la République Française pour 1'Education a été celui qui a préparé le programme et les principales questions à examiner. L'influence de ce congrès sur l'enseignement des LV sera extraordinaire et ses principales conclusions, qui élevèrent la méthodologie active, seront considérées comme incontestables. C'est alors que cette méthodologie prédominera dans presque tous les pays développés durant des décennies, faisant preuve de plus de pragmatisme, permettant l'utilisation de la LM en classe et l'assouplissement du vocabulaire, de l'oral avec l'écrit, de la grammaire, etc.

Même s'il s'est avéré que la méthodologie active n'a pas supposé, dans la façon de concevoir l'enseignement du français en Espagne, une transformation radicale par rapport à la méthodologie directe, elle aura permis d'importants changements; changements non drastiques certes, mais significatifs et expliquant une évolution difficile mais évidente. L'avènement des méthodes actives dans l'enseignement en Espagne aura donc entraîné la première tentative sérieuse de moderniser les méthodologies précédentes et de placer l'Espagne à la hauteur des autres pays européens dans l'enseignement des LV. Il paraît donc évident que sans cette étape oubliée, les méthodologies qui suivent n'auraient pas été possibles (Morales Gil, 2007).

Avec l'arrivée de la "révolution » de l'image et du son, la méthodologie audio-visuelle du $\mathrm{XX}^{\mathrm{e}}$ siècle dans les années 60 et 70 , reposant sur la linguistique structurale et le béhaviorisme, a également dominé l'évolution didactique du FLE en Espagne. Elle privilégie l'oral, accorde une grande importance aux dialogues conçus selon une progression grammaticale et lexicale, et refuse la traduction et le recours à la LM. Ainsi que le souligne Fernández Fraile, l'auteur représentatif des méthodes audio-visuelles en Espagne est Grandía Mateu :

Destaca en cualquier caso la figura de Luis Grandía Mateu, introductor e impulsor en España de los métodos audiovisuales, creando un Centro de Lingüística (en Granada), que en el que se elaborán innumerables manuales, así como de obras teóricas que permiten hablar de un método audiovisual con características propias (Fernández Fraile, 2005: 33-34).

Par ailleurs, les cours audio-visuels ont ainsi transformé les pratiques pédagogiques et ce jusque dans les années 80 , moment où l'approche communicative a fait son apparition, devenant à son tour dominante dans l'enseignement des langues en Espagne et, en particulier, celle du FLE, ouvrant la voie aux documents dits «authentiques ». Son introduction renouvelle la conception du processus d'E/A d'une langue. En ce sens, un concept novateur apparaît : la « compétence communicative » (Hymes, 1972) avec pour objectif principal d'apprendre à communiquer aussi bien à l'oral qu'à l'écrit en LE, grâce à un enseignement plus fonctionnel, centré sur l'apprenant, axé sur la spontanéité de la prise de parole et la comparaison entre la culture de la LM et celle de la LE, etc. 
L'apparition de la perspective actionnelle en 2001 ira au-delà de l'approche communicative en imposant le paradigme de l'action par l'intermédiaire de "tâches " proches de la vie quotidienne des apprenants. Il ne s'agit donc plus de communiquer pour parler avec autrui mais aussi pour agir avec lui : en somme, communiquer et agir avec les autres dans le monde. Pour Puren (2004), cette orientation didactique est insuffisante et c'est la raison pour laquelle il propose de l'appeler la «perspective co-actionnelle » mettant en relief les actions collectives qui se veulent communes et le but de ces actions qui se veut social.

A ce stade-là, l'évolution didactique du FLE arrive à son sommet puisque pour le moment la perspective actionnelle est toujours d'actualité. Les courants méthodologiques qui ont jalonné le FLE en Espagne ont été présentés sommairement. Bien qu'il en existe d'autres, l'étude s'est limitée à évoquer les principaux courants méthodologiques du $\mathrm{XX}^{\mathrm{e}}$ siècle.

\subsection{L'influence française}

Comme l'explique Morales Gil (2007), ce fut durant la Première Semaine Didactique de la Langue Française (1957) ayant attiré de nombreux Professeurs de toute l'Espagne, que les Instructions générales pour l'Enseignement des LV (approuvées par le Ministère français en 1950) ont été travaillées selon les lignes directrices du Centre National de Documentation Pédagogique et les contenus du Mémento d'Usage des Professeurs et Elèves de Lettres et Grammaire et ceux de l'Institut Pédagogique National. En parallèle, et cela est fondamental, la présentation du Français Elémentaire en 1954, a influencé de nombreux professeurs espagnols de FLE. Par ailleurs, la formation des enseignants sera l'une des constantes de la politique ministérielle espagnole dans les années 50. Les professeurs de français en bénéficieront et verront leur situation s'améliorer avec la reprise en 1954 du Diplôme de Philologie moderne et son implantation progressive dans les différentes Universités espagnoles.

Cette spécialisation, tout comme de plus grandes facilités pour compléter la formation en France à travers des " lectorats », ont ainsi amélioré le niveau des connaissances linguistiques et culturelles des futurs enseignants de FLE en Espagne. Par ailleurs, en réponse à la loi dite « Ordenación de la Enseñanza Media » de 1953, un regain d'intérêt pour les aspects pédagogiques et didactiques en général ainsi qu'une nouvelle exigence de formation pédagogique des enseignants ont été mis en place. Entre 1953 et 1963 la formation professionnelle de ces enseignants sera principalement guidée par de nouvelles organisations: le Centre d'Orientation Didactique (Centro de Orientación Didáctica) et l'Ecole de Formation des Enseignants de l'Education Secondaire (Escuela de Formación del Profesorado de Enseñanza Media). De plus, durant ces années, les séjours d'enseignants à l'étranger étaient fréquents, notamment en France, où se propageaient abondamment les nouveautés méthodologiques sur l'enseignement du français. Les influences importées trouveront ainsi un écho particulier en Espagne, à travers des réunions d'enseignants du Secondaire, qui commencèrent à s'organiser, sous la direction du Centre d'Orientation Didactique, contribuant ainsi à réformer la formation au professorat. Les enseignants seront les principaux protagonistes de toute cette évolution méthodologique. Les uns propageront les nouveautés méthodologiques à d'autres collègues, les autres se joindront à ces nouvelles connaissances et pratiques. 
Certes, le Ministère espagnol et sa politique contribuèrent beaucoup à ces changements, mais ce sera principalement le corps enseignant (surtout au lycée) qui maintiendra sa posture, en même temps qu'il voyagera et se mettra à jour, notamment en France, contribuant ainsi à une dynamique réelle du changement méthodologique.

\section{L'ELE en France}

Comme pour la didactique du FLE en Espagne, des recherches intéressantes ont été réalisées sur l'évolution didactique de l'ELE en France. Les auteurs ayant travaillé cette thématique sont principalement Puren $(1984,1988,1999)$ et Lenoir (2001, 2008, 2009), ce qui permet de mieux comprendre le cheminement de la didactique de l'espagnol dans l'enseignement en France, qui est longtemps demeurée en marge de la méthodologie audio-visuelle et de l'approche communicative. Chemin faisant, une méthodologie singulière caractérise la langue espagnole en France puisqu'elle a dû au fil du temps s'adapter aux préconisations du CECRL (Conseil de l'Europe, 2001).

\subsection{L'évolution méthodologique atypique}

La méthodologie traditionnelle en France s'étendra sur plus de trois siècles, prenant des formes différentes tout au long de son évolution, et ce n'est qu'au début du $\mathrm{XX}^{\mathrm{e}}$ siècle que la méthodologie directe s'imposera officiellement dans l'enseignement secondaire français. Par la suite, la méthodologie active, connue aussi en France sous les appellations de "méthodologie éclectique " ou encore " méthodologie mixte " (Puren, 1988 : 143), fera son entrée dès 1920. C'est d'ailleurs cette dernière qui jouera un rôle fondamental dans la tradition didactique en espagnol. Comme l'explique Lenoir (2009: 119) :

Dans sa thèse sur l'histoire de l'enseignement de l'espagnol, Christian Puren présente la « méthodologie officielle d'espagnol » comme une méthodologie éclectique qui répond clairement aux critères de définition de la Méthodologie Active. Dans cette recherche soutenue en 1984, l'auteur met très nettement en évidence que la durée de vie de la «MA hispanique » va bien au-delà des limites qu'a connues la Méthodologie Active pour les autres langues ; il va jusqu'à affirmer alors que cette méthode née dans les années 1920 « a de beaux jours devant elle ».

Cet auteur confirmera en effet dans sa thèse l'étude de Puren en démontrant que la méthodologie active d'espagnol se maintiendra au-delà des années 60 , s'étendant ainsi sur toute la deuxième moitié du $\mathrm{XX}^{\mathrm{e}}$ siècle, contrairement aux autres LE en France, qui suivront l'évolution progressive de la didactique des langues en général.

C'est ainsi que dès l'arrivée de la méthodologie audio-visuelle sur le «marché didactique » un important clivage éclata, comme l'explique Puren (1999), entre les différentes didactiques des LE dans l'enseignement français, à part celle du FLE qui avait déjà adopté l'audio-visuel quelques années auparavant. A partir de ce moment, les anglicistes qui voulaient conserver leur statut de "langue internationale » s'approprièrent immédiatement la méthodologie audio-visuelle. Les germanistes, en 
rivalité avec les anglicistes, ont fait de même tout en conservant leur «méthodologie de l'excellence » axée sur une forte présence grammaticale. Cette double stratégie ne s'avèrera guère bénéfique. Les hispanistes, quant à eux, loin d'être en rivalité avec la langue internationale ont choisi de s'en différencier, privilégiant la langue de la culture. D'où le positionnement différent des deux didactiques : « les anglicistes sacrifiant la culture à la langue, les hispanistes sacrifiant la langue à la culture » (Puren, 1999 : 12). La position des hispanistes français s'explique aussi par le fait qu'à cette époque l'Espagne était sous l'emprise du franquisme, et que jusqu'à la disparition de Franco en 1975 la communication avec cette Espagne totalitaire n'était ni envisagée ni souhaitée.

Comme l'indique à juste titre Lenoir (2009 : 8), les LE en France s'orienteront de manière différente :

C'est le début d'une sorte d'histoires parallèles : l'anglais ou l'allemand connaîtront les différentes étapes de l'évolution de la didactique des langues étrangères (DLE), se disputant le « créneau » de la LV1 dans les collèges et les lycées, tandis que la didactique de l'espagnol évoluera seule, poursuivant la tradition de la MA tout en occupant une place de plus en plus grande comme seconde langue vivante dans l'enseignement scolaire.

Le cheminement adopté par la pédagogie de l'ELE en France ne sera pas sans conséquences aussi bien positives que négatives. D'une part, la position s'est révélée judicieuse par rapport aux autres didactiques et parfois même en avance sur elles, en ce sens qu'elle a engendré une meilleure motivation chez les apprenants, un lien fort entre langue et culture, et la revalorisation de la culture. D'autre part, en refusant la méthodologie audio-visuelle, combinant les supports auditifs et visuels dans l'acception large du terme, la pédagogie de l'ELE a opté pour une faible intégration de l'audio en faveur du texte littéraire et une importante intégration du visuel, ce qui a entraîné des effets négatifs comme des carences en compréhension orale, en expression écrite, en prononciation, et la prééminence de la communication académique, ou des faiblesses dans l'apprentissage de la langue en termes de lexique et de grammaire (Puren, 1999). A cela s'ajoutent d'autres inconvénients :

Un certain nombre d'effets négatifs sont liés simplement au fait que les hispanistes se sont retrouvés seuls sur une didactique originale. En restant volontairement en dehors de l'évolution des autres langues et de la didactique en général, ils ont fini par développer une sorte de "stratégie de la tour d'ivoire », basée sur la certitude un peu perverse qu'ils sont les meilleurs parce qu'ils sont les seuls... Ils ont eu tendance, mécaniquement, à « durcir» leurs spécificités, à en faire une doctrine. Pour poursuivre la comparaison entre les deux traditions les plus opposées, on pourrait dire que les anglicistes ont eu tendance à dogmatiser à partir de théorie de référence [...], et que les hispanistes ont dogmatisé à partir de pratiques de référence. $\mathrm{La}$ " tradition didactique ", en espagnol se présente d'abord comme une tradition méthodologique, comme on le voit dans les instructions officielles d'espagnol, avec tous les effets négatifs que l'on connaît dans la formation initiale des stagiaires (Puren, 1999 : 14-15). 
Selon l'auteur, l'espagnol s'est alors mis à l'écart de l'évolution des autres LE et de celle de la didactique des langues au sens large, développant ainsi, pour reprendre l'expression de Puren, une sorte de "stratégie de tour d'ivoire » qui à l'usage s'est révélée néfaste, puisqu'elle a privilégié l'accès à la LE à travers des textes culturels, en considérant que c'est au fur et à mesure que les apprenants parlent sur ce type de support, qu'ils finissent par parler la langue. Or, la formation en commentaire de textes, encore d'actualité de nos jours, est loin d'être la méthode la plus efficace. Mais concrètement, que s'est-il donc passé pour l'ELE en France au-delà des années 60 ?

La thèse doctorale de Lenoir (2009) apporte des éléments de réponses concises à cette problématique sur une période de temps comprise entre 1970 et 2007. Sa recherche sur l'évolution méthodologique de l'espagnol, qualifiée de singulière, a montré que la méthodologie active qui avait dominé l'apprentissage de l'espagnol en France durant toute la deuxième moitié du $\mathrm{XX}^{\mathrm{e}}$ siècle, avait délibérément mis en marge la méthodologie audio-visuelle et l'approche communicative dont l'influence sur l'enseignement hispanique avait été minime voire insignifiante. Le chercheur a donc voulu d'une part savoir les raisons pour lesquelles cette méthodologie active avait pris le monopole sur les autres méthodologies, et d'autre part en mesurer les effets au moment même où apparaissent les recommandations du CECRL pour l'enseignement de toutes les LE, avec à la carte une nouveauté européenne : la perspective actionnelle.

\subsection{L' « ellipse méthodologique »}

Pour étayer sa thèse, Lenoir (2009 : 10) emploie un concept personnel pour mieux expliquer l'évolution atypique de la didactique de l'ELE en France, celui du concept de 1' « ellipse méthodologique » allant même jusqu'à parler de 1'apparition d'un « Objet Didactique Non Identifié » dans le domaine de la didactique (Lenoir, 2009 : 119). Il faut dire aussi que la méthodologie active et/ou éclectique de l'espagnol, évoquée précédemment, n'avait jamais fait l'objet d'investigations profondes contrairement aux autres méthodologies plus connues du grand public. Selon Lenoir (2009: 120) :

Il s'agissait en 1920 de réaliser une sorte de compromis méthodologique - le terme est souvent employé par les méthodologues du début du XX ${ }^{\mathrm{e}}$ siècle -, la ME constituant une sorte d' « ODNI » (Objet Didactique Non Identifié), puisqu'elle associait dès l'origine plusieurs aspects de la MT (ou de grammaire-traduction) et de la MD.

Voilà pourquoi cette méthodologie, empruntant ses procédés aussi bien à la méthodologie traditionnelle qu'à la méthodologie directe, a longtemps été considérée comme un cas particulier en France. Méthodologie dominante de l'espagnol dans l'enseignement scolaire, ce schéma de classe s'est perpétué jusqu'à nos jours :

La conception des objectifs et de leur articulation est donc demeurée quasiment inchangée en espagnol entre les années 1920 et 2002, date de la publication de l'instruction pour la classe de seconde. On peut faire le même constat en ce qui concerne la pédagogie : au cours de la quasi-totalité du $\mathrm{XX}^{\mathrm{e}}$ siècle, le schéma de classe est lui aussi resté très stable (Lenoir, 2009 : 129). 
Comme le précise Lenoir (2009: 140), un long « décrochage » didactique caractérise l'ELE en France qui s'est traduit par une " ellipse méthodologique » mettant de côté la méthodologie audio-visuelle et l'approche communicative. C'est ainsi qu'une évolution singulière de l'enseignement de cette langue s'est longtemps fondé sur une méthodologie active et/ou éclectique qui a modelé jusqu'ici le processus d'E/A de l'ELE en France. Nonobstant, il est certain que la parution du CECRL a transformé la didactique de l'espagnol et des autres LE en France et en Europe.

\section{Conclusion}

L'étude montre que l'évolution didactique du FLE et de l'ELE, dans un contexte particulier, est unique en son genre, et ne suit pas forcément les grandes lignes des méthodologies de la DLE qui se sont succédé dans le temps. En Espagne, ce sont pourtant les méthodologies les plus représentatives qui ont marqué l'évolution de la didactique du FLE. Cela met en évidence, par conséquent, une évolution méthodologique progressive qui connaîtra les différentes étapes de celle de la DLE dans sa perspective générale. En France, une méthodologie singulière caractérise l'ELE, le dotant d'une évolution didactique atypique. C'est la méthodologie active qui aura pris le monopole sur la méthodologie audio-visuelle et l'approche communicative et ce jusqu'à l'apparition du CECRL. Dès lors, la perspective actionnelle qui en découlera permettra d'ouvrir de nouveaux parcours didactiques en harmonisant toutes les langues à l'échelle nationale et internationale.

\section{Références bibliographiques}

Barthélémy, Fabrice (2007): Professeur de FLE : Historique, enjeux et perspective. Paris: Hachette.

Barthélémy, Fabrice; Groux, Dominique; Porcher, Louis (2013): Le français langue étrangère. Paris: L'Harmattan.

Bourdieu, Pierre (1979): La distinction. Paris: Minuit.

Calle Carabias, Quintín (1990): La enseñanza de idiomas en España. Malaga: UP.

Cassany, Daniel (2009): “¿Cuál el estado actual de la didáctica del ELE? ¿Qué ha pasado durante estos años? ¿Qué no ha pasado y debería haber pasado? ¿Cuáles son las perspectivas de futuro?". MarcoELE. Revista de Didáctica Español como Lengua Extranjera, 9: 1-21. Disponible sur http://marcoele.com/descargas/9/veinticinco.pdf

Cestero Mancera, Ana María (2006): Lingüistica aplicada a la enseñanza del español como lengua extranjera: desarrollos recientes. Alcalá de Henares: Universidad de Alcalá de Henares.

Conseil de l'Europe (1998): "Recommandation 1383 - Diversification linguistique et réponse du Comité des Ministre". Strasbourg : Conseil de l'Europe. Disponible sur : http://assembly.coe.int/nw/xml/XRef/Xref-XML2HTML-FR.asp?fileid=16644\&lang=FR （consulté le 11.02.2014).

Conseil de l'Europe (2001): Cadre Européen Commun de Référence pour les Langues. Paris: Didier.

Cuq, Jean-Pierre et Gruca, Isabelle (2003): Cours de didactique du français langue étrangère et seconde. Saint-Martin-d'Hères (Isère): Presses Universitaires de Grenoble. 
Fernández Fraile, María Eugenia (1995). La enseñanza/aprendizaje del francés como lengua extranjera en España (1767-1936): objetivos, contenidos y procedimientos. Granada: Universidad.

Fernández Fraile, María Eugenia; Suso López, Javier (1999): La enseñanza del francés en España (1767-1936). Estudio histórico: objetivos, contenidos, procedimientos. Granada: Método Ediciones.

Fernández Fraile, María Eugenia (2005): "La institucionalización de la enseñanza del francés como materia escolar en el siglo XX: planes de estudio y orientaciones oficiales". Revista interuniversitaria de formación del profesorado 53: 19-46.

García Bascuñana, José Francisco (2005): "Materiales para la enseñanza del francés en España: aproximación a los manuales publicados entre los Siglos XVI y XX". Revista Interuniversitaria de Formación del Profesorado 19 (2): 129-144.

García Bascuñana, José Francisco (2008): “¿Con qué libros se aprendía francés en España en 1808?’. Anales de Filología Francesa 16: 73-85.

Hymes, Dell Hathaway (1972): “On communicative competence”, en Sociolinguistics, J.B. Pride et J. Holmes (Eds.), pp. 269-293. London: Penguin.

Lenoir, Pascal (2001): "Former en IUFM à une utilisation critique des matériels didactiques d'espagnol publiés en France", en Actes des Journées angevines sur la didactique de l'espagnol, 1: 17-29.

Lenoir, Pascal (2008): "Vers la fin d'un demi-siècle d' " ellipse » méthodologique en espagnol ?". L'enseignement de l'espagnol en France : réalités et perspectives. Valenciennes : Presses Universitaires de Valenciennes.

Lenoir, Pascal (2009): De l'ellipse méthodologique à la perspective actionnelle : la didactique scolaire de l'espagnol entre tradition et innovation (1970-2007). Thèse de Doctorat. Université Jean Monnet de Saint-Étienne.

Lépinette, Brigitte (1997): "Deux grammaires françaises pour espagnols (XVIIe siècle): traditions grammaticales nationales et pédagogie". Linguistica e investigationes: Revue internationale de linguistique française et de linguistique générale, Tome 21, Fascicule 1: 199-239

Lépinette, Brigitte (2000): “Gramáticas del francés editadas en España durante la primera mitad del siglo XIX”. Quaderns de filología. Estudis lingüístics, 5, (Ejemplar dedicado a: Aprendizaje y enseñanza de una segunda lengua): 177-196.

Lépinette, Brigitte (2006): "La didáctica lingüística en Francia y en España ( $1^{\text {a }}$ mitad del siglo XIX). Convergencias e influencias", en La cultura del otro: español en Francia, francés en España, M. Bruña Cuevas, M. de Gracia Caballos Bejano, I. Illanes Ortega, C. Ramírez Gómez, A. Raventós Barangé (Coords), pp. 1052-1066. Sevilla: Universidad de Sevilla.

Morales Gil, Francisco José (2003): La evolución metodológica de la enseñanza oficial del francés en España: 1936-1970. Tesis doctoral. Huelva: Universidad de Huelva.

Morales Gil, Francisco José (2007): “La difusión de la metodología activa en la enseñanza de los idiomas en España: El caso del francés". Revista Internacional de Investigación e Innovación Educativa vol 9: 237-251.

Morales Gil, Francisco José (2009): “La enseñanza de idiomas en España en la frontera de los años 30: las ilusiones perdidas". Documents pour l'histoire du français langue étrangère ou seconde 42: 231-248.

Porcher, Louis (1987): Champs de signes. États de la diffusion du français langue étrangère. Paris: Crédif/Didier.

Puren, Christian (1984): La didactique de l'espagnol en France : évolution historique, situa- 
tion actuelle et nouvelles perspectives. Thèse de doctorat. Université de Toulouse-le-Mirail.

Puren, Christian (1988): Histoire des méthodologies de l'enseignement des langues. Paris : Nathan / CLE International.

Puren, Christian (1999): "La tradition didactique en espagnol face aux évolutions actuelles". Didactique de l'espagnol (1): langue, culture et stratégies de formation - Actes des «Journées angevines ». Université de Nantes, IUFM des Pays de la Loire, 4: 11-16.

Puren, Christian (2004): "Recherche et pratiques pédagogiques en langues de spécialité". Cahiers de l'Apliut. Vol XXVIII, 1: 10-26.

Rodríguez Seara, Ana (2001): “L'évolution des méthodologies dans l'enseignement du français langue étrangère depuis la méthodologie traditionnelle jusqu' à nos jours". Cuadernos del Marqués de San Adrián: revista de humanidades 1: 139-161.

Suso López, Javier (2005): "La esperanza del francés en España". Revista interuniversitaria de formación del profesorado 53: 15-18.

Urbano Marchi, Brigitte (2003): La enseñanza/aprendizaje del francés como lengua extranjera en España durante la época franquista (1938-1970). Tesis doctoral. Universidad de Granada. 